F. PÁPAI, S. ADHIKARI, B. WANG

\title{
ESTIMATION OF MODAL DAMPINGS FOR UNMEASURED MODES
}

\section{ABSTRACT}

Damping effects are of great interest for structural analysis and evaluations. Structural modal damping characteristics can be obtained from experiments. This paper introduces new possibilities for the modelling of the damping of a dynamic system with classical normal modes and provides an overview of the known methods for formulating a damping matrix base with experimental modal damping values. The proposed method offers an opportunity to extrapolate modal damping values for unmeasured modes by a regression method based on the measured modal properties. The points of view on the choice of an analytical form for damping regression functions are examined. An analytical form of regression functions can be chosen as the modal decay rate versus the square of the frequency or the modal damping ratio versus the frequency. Damping regressions can be performed based on a group of typical vibration modes, such as bending, torsion and lateral, symmetrical or anti-symmetrical modes. The regression data obtained for the damping constants can then be applied in a finite element model for further structural analysis.

\section{INTRODUCTION}

The equation of motion of a viscously damped system is expressed by
(1) $\quad \mathbf{Z}(\lambda)=\lambda^{2} \mathbf{M}+\lambda \mathbf{C}+\mathbf{K}$

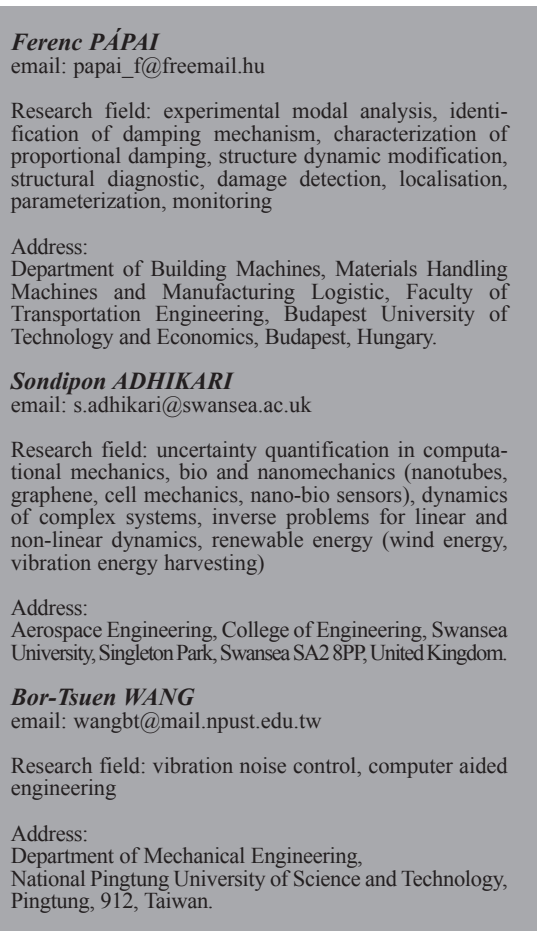

KEY WORDS

- Modal damping ratio,

- Modal decay rate,

- Regression,

- Damping matrix. 
The eigenvalues $\lambda_{i}=\delta_{i}+j v_{i}, \quad(i=1,2, \ldots, 2 n)$ are complex quantities. $\delta_{i}$ are damping, and $v_{i}$ is the damped natural frequency. It is known that for symmetrical undamped systems, the $\lambda_{i}, \quad(i=1,2, \ldots, 2 n)$ eigenvalues of the $\mathbf{Z}(\lambda)=\lambda^{2} \mathbf{M}+\mathbf{K}$ system matrix are pure imaginary quantities, and due to the $\lambda_{n+i}=-\lambda_{i}(i=1,2, \ldots, n) \Rightarrow \Omega_{i}^{2}:=-\lambda_{i}^{2}, \quad(i=1,2, \ldots, n)$, there are $n$ eigenvectors $\boldsymbol{\varphi}_{i}$, which are linearly independent. They can be made for vectors with real elements by appropriate normalisation. The equivalent transformation, which is performed by a modal matrix constructed from eigenvectors $\boldsymbol{\Phi}=\left[\boldsymbol{\varphi}_{i}\right]$, simultaneously diagonalizes $\mathbf{M}$, K. Such modal shapes of undamped systems are called normal modes or classical normal modes in the literature. In the case of normal modes all the displacement coordinates of the structure reach the extreme position at the same time (pure phase / outphase; in other words, a standing wave in a modal shape).

The concept of decaying systems of classical normal modes was introduced by Lord Rayleigh (1877), who proved that classical modes can exist in damped systems too. Such a case is, for example, the so-called proportional damping, where the damping matrix $\mathbf{C}$ is a linear combination of $\mathbf{M}$ and $\mathbf{K}$, that is:

\section{$\mathbf{C}=\alpha \mathbf{M}+\beta \mathbf{K} \quad($ Rayleigh damping $)$}

where the $\alpha, \beta$ material pendants (usually real) are scalar constants.

Caughey and O'Kelly (1965) [1] proved that a necessary and sufficient condition for the existence of classical normal modes is to satisfy

$$
\mathbf{C M}^{-1} \mathbf{K}=\mathbf{K M}^{-1} \mathbf{C}
$$

the so-called commutative relationship $\left(\mathbf{M}^{-1} \mathbf{C}\right) \cdot\left(\mathbf{M}^{-1} \mathbf{K}\right)=\left(\mathbf{M}^{-1} \mathbf{K}\right) \cdot\left(\mathbf{M}^{-1} \mathbf{C}\right)$. If the commutative relationship shown in Eq. (4) is satisfied for the coefficient matrices of system Eq. (1), the real modal coordinate transformation

\section{$\mathbf{x}=\boldsymbol{\Phi q}$}

can be performed. In this modal space the equation of the motion of a viscously damped system is expressed by

$$
\boldsymbol{\Phi}^{T} \mathbf{M} \boldsymbol{\Phi} \ddot{\mathbf{q}}+\boldsymbol{\Phi}^{T} \mathbf{C} \boldsymbol{\Phi} \mathbf{q}+\boldsymbol{\Phi}^{T} \mathbf{K} \boldsymbol{\Phi} \mathbf{q}=\boldsymbol{\Phi}^{T} \mathbf{f}(t)
$$

where

$$
\boldsymbol{\Phi}^{T} \mathbf{M} \boldsymbol{\Phi}=\mathbf{E}, \boldsymbol{\Phi}^{T} \mathbf{C} \boldsymbol{\Phi}=\left\langle-2 \delta_{i}\right\rangle, \boldsymbol{\Phi}^{T} \mathbf{K} \boldsymbol{\Phi}=\left\langle\Omega_{i}^{2}\right\rangle=\mathbf{\Omega}^{2}, \boldsymbol{\Phi}^{T} \mathbf{f}(t)=\boldsymbol{\Gamma}(t)
$$

$$
\text { (6c) }
$$

$(6 e)$

notation $\langle$.$\rangle means a diagonal matrix.$

Caughey (1960) [2] verified that these normal modes of a damped system are equivalent to the modes of an undamped system. "In the case of a damped linear system, classical normal modes exist if and only if the damping matrix is diagonalized by the same transformation, which makes the undamped system diagonal." He formulated a sufficient condition for coefficient matrices: "In damped systems, the condition for classical normal modes is that the product $\mathbf{M}^{-1} \mathbf{C}$ can be expressed as the power of product $\mathbf{M}^{-1} \mathbf{K}$, in the form of the following series:"

$$
\mathbf{M}^{-1} \mathbf{C}=\sum_{q=1}^{\infty} \sum_{p=0}^{n-1} a_{p q}\left(\mathbf{M}^{-1} \mathbf{K}\right)^{\frac{p}{n}}
$$

He proposed the following expression for the possible analytical form of a damping matrix that satisfies the condition mentioned above:

$$
\mathbf{C}=\mathbf{M} \sum_{p=0}^{n-1} a_{p}\left(\mathbf{M}^{-1} \mathbf{K}\right)^{p}
$$

This form is called the Caughey series in the literature. The damping matrix formed by Eq. (8) is also known as the proportional damping matrix after Rayleigh.

The traditional Rayleigh damping in Eq. (3) is a special case of the expression in Eq. (8), because with the substitution $a_{0}=\alpha, a_{1}=\beta$, $a_{2}=a_{3}=\ldots=a_{\mathrm{n}-1}=0$, we have:

$$
\mathbf{C}=\mathbf{M} a_{0}\left(\mathbf{M}^{-1} \mathbf{K}\right)^{0}+\mathbf{M} a_{1}\left(\mathbf{M}^{-1} \mathbf{K}\right)^{1}=a_{0} \mathbf{M}+a_{1} \mathbf{K} .
$$

Fawzy (1977) [3] gave the following analytical form for a damping matrix: $\mathbf{C}=\mathbf{M} \sum_{q=1}^{\infty} \sum_{p=0}^{t+2} a_{p q}\left(\left(\mathbf{M}^{-1} \mathbf{K}\right)^{\bar{q}}\right)^{p+q}$, which satisfied the condition in Eq. (4). Sas et al. (1998) [4] composed the commutative relationship in the form of $\left(\mathbf{M}^{-1} \mathbf{C}\right)^{p}\left(\mathbf{M}^{-1} \mathbf{K}\right)^{q}=\left(\mathbf{M}^{-1} \mathbf{K}\right)^{q}\left(\mathbf{M}^{-1} \mathbf{C}\right)^{p}$.

Adhikari (2000) [5] suggested a further generalization for a proportional damping matrix. He proved that if the damping matrix can be expressed as

$$
\begin{aligned}
& \mathbf{C}=\mathbf{M} f_{1}\left(\mathbf{M}^{-1} \mathbf{K}\right), \\
& \mathbf{C}=\mathbf{K} f_{2}\left(\mathbf{K}^{-1} \mathbf{M}\right), \\
& \mathbf{C}=\mathbf{M} f_{1}\left(\mathbf{M}^{-1} \mathbf{K}, \mathbf{K}^{-1} \mathbf{M}\right)+\mathbf{K} f_{2}\left(\mathbf{M}^{-1} \mathbf{K}, \mathbf{K}^{-1} \mathbf{M}\right), \\
& \mathbf{C}=f_{3}\left(\mathbf{K M}^{-1}, \mathbf{M} \mathbf{K}^{-1}\right) \mathbf{M}+f_{4}\left(\mathbf{K M}^{-1}, \mathbf{M} \mathbf{K}^{-1}\right) \mathbf{K},
\end{aligned}
$$

where $f_{1}(), f_{1}(), f_{3}(), f_{4}()$ are analytical functions, the system possesses classical normal modes.

\subsection{The relationship between modal characteristics and the damping matrix}

Let us study Adhikari's relationship as given by Eq. (10)

$$
\mathbf{C}=\mathbf{M} f_{1}\left(\mathbf{M}^{-1} \mathbf{K}\right) \text {. }
$$


In this relationship replace $\mathbf{M}, \mathbf{M}^{-1}, \mathbf{K}$ with their spectral decomposition to express the modal matrices from Eqs. (6b) and (6c)

$$
\mathbf{C}=\boldsymbol{\Phi}^{-T} \boldsymbol{\Phi}^{-1} f_{1}\left(\boldsymbol{\Phi} \boldsymbol{\Phi}^{T} \boldsymbol{\Phi}^{-T} \boldsymbol{\Omega}^{2} \boldsymbol{\Phi}^{-1}\right)=\boldsymbol{\Phi}^{-T} \boldsymbol{\Phi}^{-1} f_{1}\left(\boldsymbol{\Phi} \boldsymbol{\Omega}^{2} \boldsymbol{\Phi}^{-1}\right),
$$

and then the specified $\mathbf{C}$ damping matrix transforms into the modal space

$$
\boldsymbol{\Phi}^{T} \mathbf{C} \boldsymbol{\Phi}=\boldsymbol{\Phi}^{T} \boldsymbol{\Phi}^{-T} \boldsymbol{\Phi}^{-1} f_{1}\left(\boldsymbol{\Phi} \boldsymbol{\Omega}^{2} \boldsymbol{\Phi}^{-1}\right) \boldsymbol{\Phi}
$$

Bring forward matrices $\boldsymbol{\Phi}, \boldsymbol{\Phi}^{-1}$ from this spectral decomposition $f_{1}\left(\boldsymbol{\Phi} \boldsymbol{\Omega}^{2} \boldsymbol{\Phi}^{-1}\right)$ [6, p. 249],

$$
\boldsymbol{\Phi}^{T} \mathbf{C} \boldsymbol{\Phi}=\boldsymbol{\Phi}^{T} \boldsymbol{\Phi}^{-T} \boldsymbol{\Phi}^{-1} \boldsymbol{\Phi} f_{1}\left(\boldsymbol{\Omega}^{2}\right) \boldsymbol{\Phi}^{-1} \boldsymbol{\Phi}=f_{1}\left(\boldsymbol{\Omega}^{2}\right) .
$$

Substituting the modal damping Eq.(6 c) $\boldsymbol{\Phi}^{T} \mathbf{C} \boldsymbol{\Phi}=\left\langle-2 \delta_{i}\right\rangle$ relationship into Eq. (17), we finally get

$$
\left\langle-2 \delta_{i}\right\rangle=f_{1}\left(\mathbf{\Omega}^{2}\right)
$$

as a simple equation. This expression means the following:

„Modal dampings $-2 \delta_{i}$ of symmetrical, single eigenvalued systems with classical normal modes are equal to the return values of a generalized Rayleigh damping matrix generator function $f_{1}($.$) at undamped eigenfrequencysquare \Omega_{i}^{2}$ locations."

In the case of classic Rayleigh damping the function in Eq. (18) has been known for a long time: $f_{1}\left(\boldsymbol{\Omega}^{2}\right)=a_{0} \mathbf{E}+a_{1} \cdot \mathbf{\Omega}^{2}$, which is seen in Fig. 1.

The generator function of the Caughey series defined in Eq. (8) is $f_{1}\left(\boldsymbol{\Omega}^{2}\right)=\sum_{p=0}^{n-1} a_{p}\left(\boldsymbol{\Omega}^{2}\right)^{p} \quad$ or $f_{1}(\lambda)=\sum_{p=0}^{n-1} a_{p}(\lambda)^{p}$ as a scalar function.

A key objective of this paper is to exploit the generic functional relationship in Eq. (18) and investigate whether this can be used effectively to model a damping mechanism in practical engineering problems. A novel idea based on the grouping of similar modes has been proposed in this context.

\section{IDENTIFICATION OF THE PARAMETERS OF A DAMPING MECHANISM}

The relationship in Eq. (18) can be appropriate for identifying the parameters of a damping mechanism. If the regression function is known, the following procedure and possible application can be used (Fig. 2):

$$
\mathbf{C}=\mathbf{M} f_{1}\left(\mathbf{M}^{-1} \mathbf{K}\right)=\mathbf{M}\left(a_{0} \mathbf{E}+a_{1} \mathbf{M}^{-1} \mathbf{K}\right)
$$

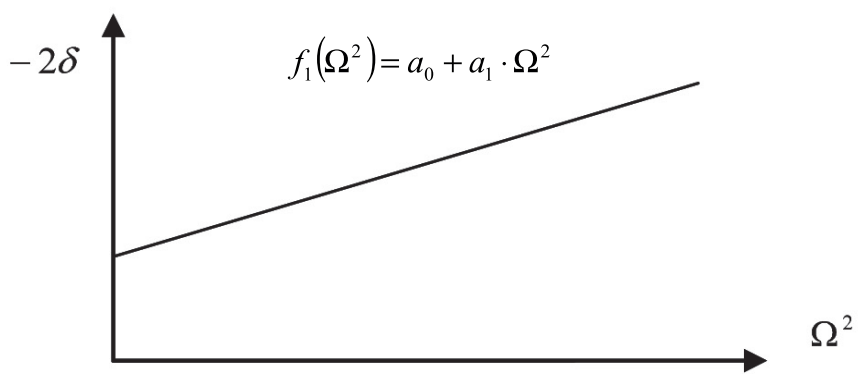

Fig. 1 Generator function of the proportional (Rayleigh) damping matrix.

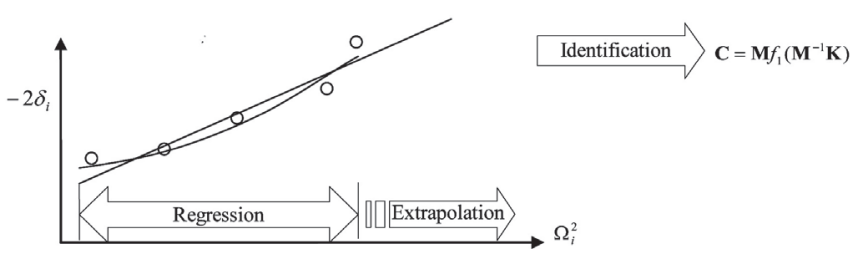

Fig. 2 Regression of generalised Rayleigh damping.

1. An experimental modal analysis is carried out to determine the eigenfrequencies and damping values in the frequency range investigated.

2. These value pairs are drawn according to a chart (Fig. 2), and then the regression function $f_{1}(\cdot)$ is determined.

3. Extrapolate the regression function $f_{1}(\cdot)$ to the outband modes (to the higher frequency range).

4. The results are available for FEM programs as damping data input.

5. The damping matrix can be written in the explicit form $\mathbf{C}=\mathbf{M} f_{1}\left(\mathbf{M}^{-1} \mathbf{K}\right)$.

The following questions or problems occur relating to regression:

a) Undamped eigenfrequencies cannot be established by experimental measurements.

b) $\mathrm{W} \mathrm{h}$ a t kind of quantities should be represented for $f_{1}\left(\Omega^{2}\right)$ a regression?

c) Is the regression function considered global in the sense that it is valid for all the modal types?

d) What should the analytical form of the regression function be?

As is well known, undamped eigenfrequencies cannot be determined by experimental measurements. The undamped system eigen- 
frequencies $\Omega_{i}$ are included in Eq. (18); however, the experimental measurements define the damped eigenfrequencies $v_{i}$. The approximation $v_{i} \approx \Omega_{i}$ is widely accepted in the case of small damped systems. For plotting the relationship between damping vs. an eigenfrequency, the $\left(\Omega_{i} ; \xi_{i}\right)$ (undamped eigenfrequency vs. Lehr damping) diagram is used in the literature, e.g., [10]-[14]. In the case of the measurement data $\left(v_{i} ; \xi_{i}\right)$ points are plotted, where the damped eigenfrequencies are $v_{i} \approx \Omega_{i}$. If the undamped eigenfrequency squares $\Omega_{i}^{2}$, as determined by FEM or any analytical methods, are available, it is advisable to use them.

As to what kind of quantities should be represented for the regression, we propose the selection of the regression function for plotting $\left(v_{i}^{2} ;-2 \delta_{i}\right)$ instead of $\left(v_{i} ; \xi_{i}\right)$, because the former is appropriate for the function defined in Eq. (18).

\section{CASE STUDIES}

In the following sections we introduce a selection of graph representations suitable for evaluating the experimental measurement results.

\subsection{Experimental investigations: I. Cantilever beam}

Measurements of the EMA (Experimental Modal Analysis) cantilever beam, SISO (Single Input - Single Output), and FRF (Frequency Response Function) were performed (Fig. 3). A schematic diagram of the experimental setup is shown in the illustration.

The excitation and response measurements were performed horizontally (xy). The total number of the measured locations was 153 . $\mathrm{N}=5$ modes were detected in the investigated frequency range $[0-200 \mathrm{~Hz}$. The LSFD (Least Squares Frequency Domain) method was applied to identify the eigenfrequencies and damping parameters [8] (Fig. 4).

The results are shown in Table $\mathbf{1 .}$

Then we composed a modal model of the structure and visualized its modal shapes. The first four bending modes and the first torsion mode appeared in the investigated frequency band. The third bending and the shape of the first torsion modes are shown in Fig. 5. The eigenvalue parameters seen in Table 1 are plotted in Fig. 6a by a traditional representation. The features of the curve can be identified with difficulty on this chart.

In the proposed representation shown in Fig. 6b, the relationship of the values presents a square root function. A regression with the analytical form $\delta_{i}=a_{1} \cdot\left(v_{i}^{2}\right)^{0}+a_{2} \cdot\left(v_{i}^{2}\right)^{1 / 2}$ was carried out, which corresponds to a linear regression on the $\left(v_{i}, \delta_{i}\right)$ values (Fig. 6c). The results of the regression for function $\delta_{i}=f_{1}\left(v_{i}^{2}\right)$ can be seen in Fig. 7, which is proper for the extrapolation of damping in an outband frequency range.

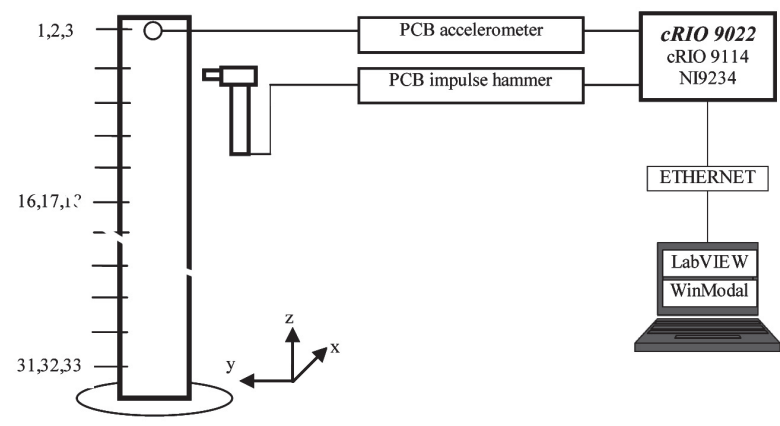

Fig. 3. Schematic representation of the experimental setup of the cantilever beam [8] (EMA, SISO, FRF measurements).

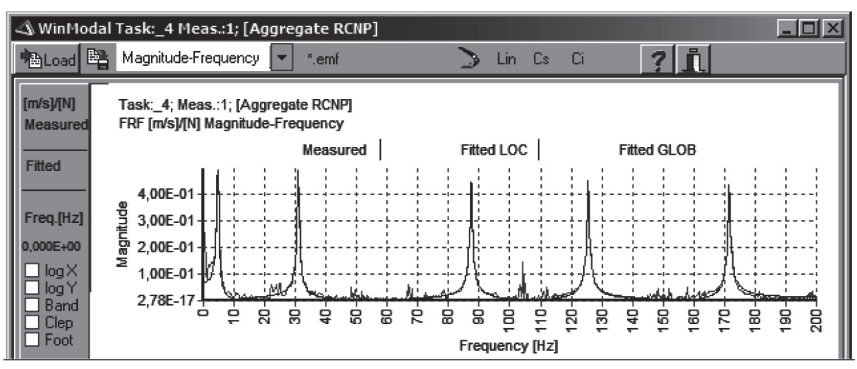

Fig. 4 LSFD regression of the measured FRF function [8].

Table 1 Eigenfrequency and damping values of the cantilever beam [8].

\begin{tabular}{|c|c|c|c|c|c|}
\hline Mode $i$ & 1 & 2 & 3 & 4 & 5 \\
\hline$f_{\mathrm{i}}[\mathrm{Hz}]$ & 4.671 & 30.93 & 87.36 & 125.5 & 171.5 \\
\hline$\xi_{\mathrm{i}}[\%]$ & 5.49 & 0.918 & 0.373 & 0.282 & 0.224 \\
\hline Type & BXZ 1 & BXZ 2 & BXZ 3 & TZ 1 & BXZ 4 \\
\hline
\end{tabular}

BXZ bending

$\mathrm{T}$ torsion

According to the experimental results, in the case of the investigated prismatic beam, the regression function $f_{1}($.$) is global for both the$ bending and torsion modes. Only one torsion mode was detected in the frequency range investigated. We think more tests should be carried out in a higher frequency band for higher torsion modes.

The problem of determining the shape of a curve in regression analysis is related to the question of whether the specific function $f_{1}($.$) is global. The global f_{1}($.$) means the function f_{1}($.$) is valid$ for all the modes.

We conducted some experimental measurements, where we found that different types of modes could have different regression functions. Some of them are shown below. 


\section{SIOVALS JOUSSILL \\ of

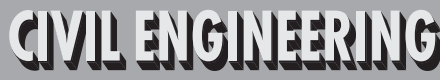

2012/4 PAGES $17-27$

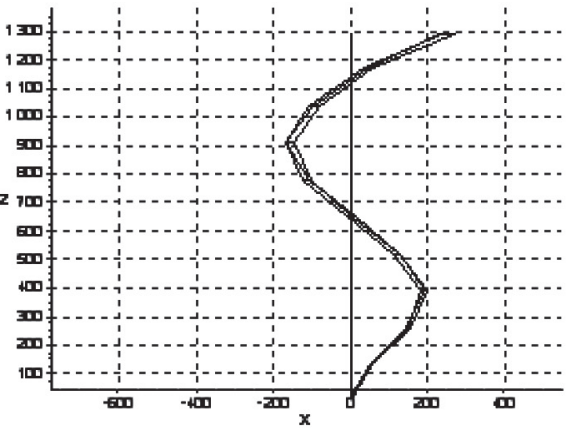

Mode: 3, BXZ 3, f=87 Hz (0.37\%)

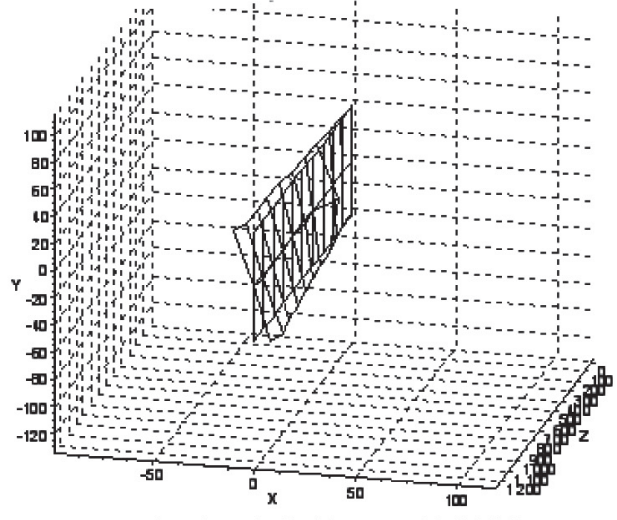

Mode: 4 , $\mathrm{T} 1 \mathrm{f}=125 \mathrm{~Hz}(0.28 \%)$

Fig. 5 Modal shapes of the cantilever beam [8].

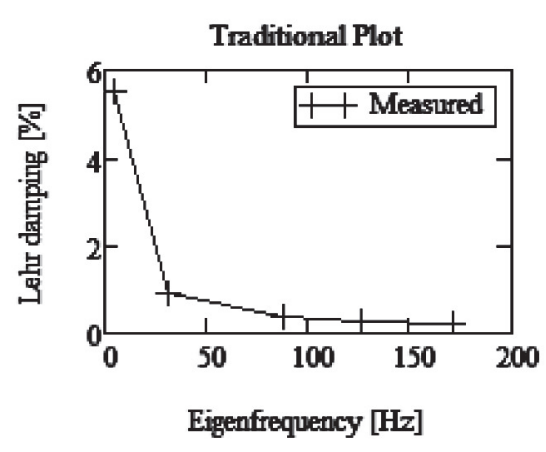

a. Traditional view

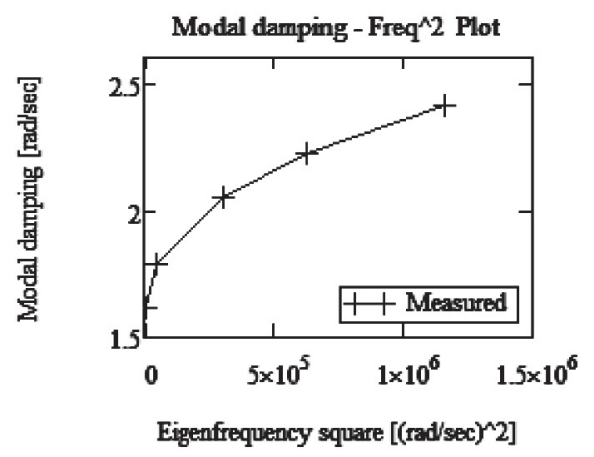

b. Proposed view

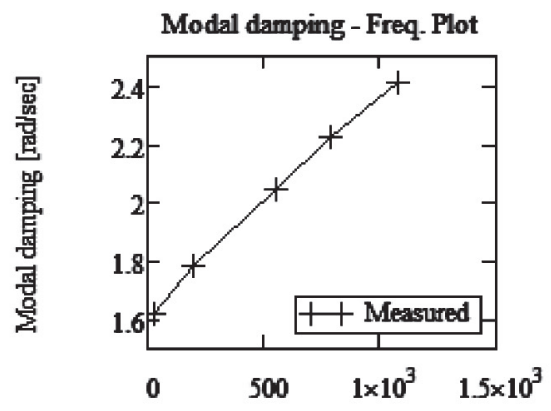

Eigenfrequency [rad/sec]

c. $\left(v_{i}, \delta_{i}\right)^{-}$, view

Fig. 6 Methods representating of the cantilever beam's eigenvalue parameters.

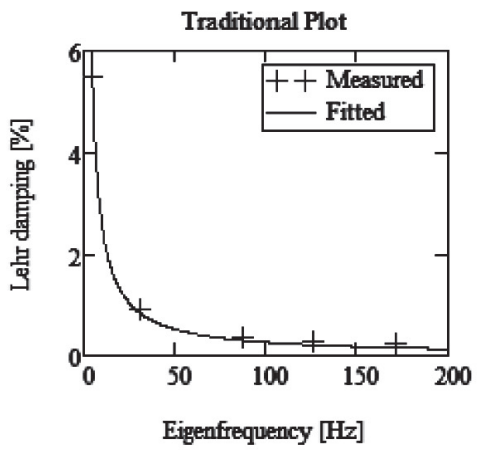

a. Traditional view

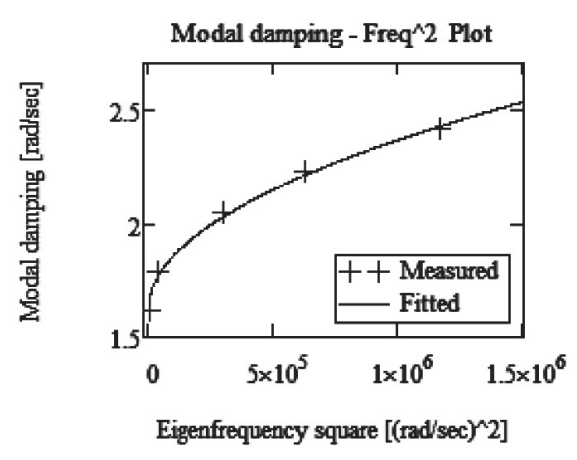

b. Proposed view

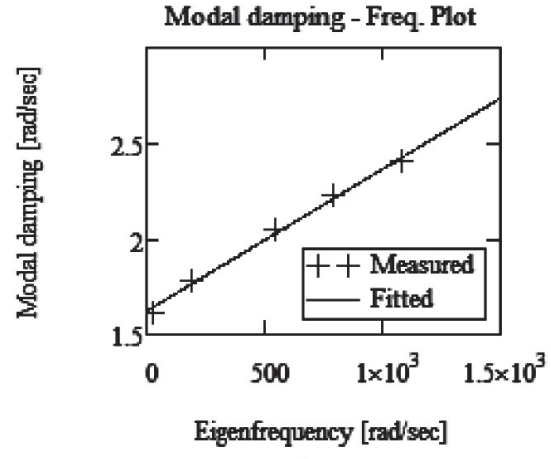

c. $\left(v_{i}, \delta_{i}\right)^{\prime}$ view

Fig. 7 Regression of the eigenvalue parameters. 


\subsection{Experimental investigation II: Damping Identification in a Clamped Plate With Slots}

In this section we consider a two-dimensional structure [7]. A schematic model of the test structure is shown in Fig. 8. This is fabricated by making slots in a mild steel rectangular plate of a $2 \mathrm{~mm}$ thickness, resulting in three-cantilever beams joined at their base by a rectangular plate. The source of the damping in this test structure is the wedged foam between the fork benches 1 and 2 .

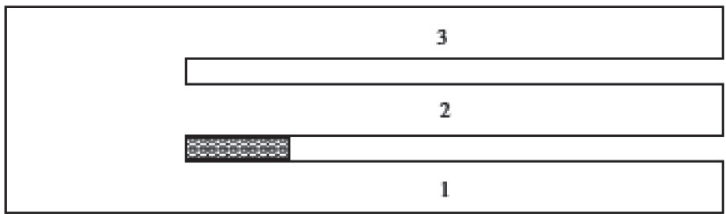

Fig. 8 Geometric shape of the plate with slots [7].

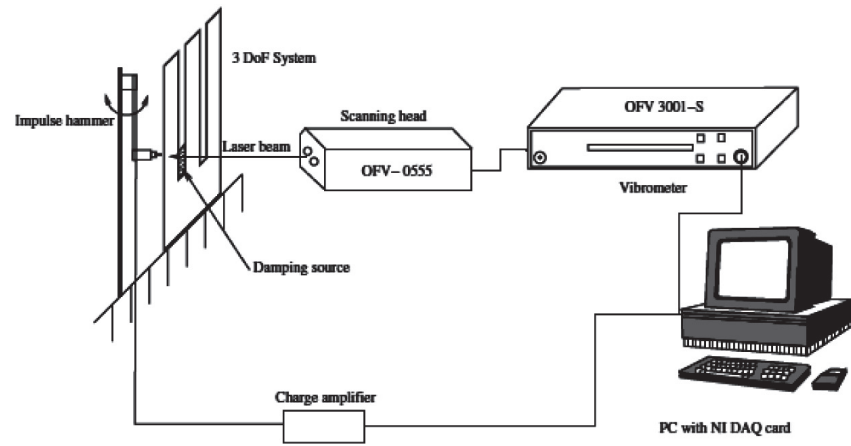

Fig. 9 Schematic representation of the experimental setup of the clamped plate with slots [7].

A schematic diagram of the test rig is shown in Fig. 9.

A classic EMA SISO FRF measurement was performed. The modal shapes from the 4th to the 9th are shown in Fig. 10. On the left side modes $4^{\text {th }}, 5^{\text {th }}$ and $6^{\text {th }}$ are the second bending, torsion and mixed modes. On the right modes $7^{\text {th }}, 8^{\text {th }}$ and $9^{\text {th }}$ are the third bending, torsion and mixed modes respectively.
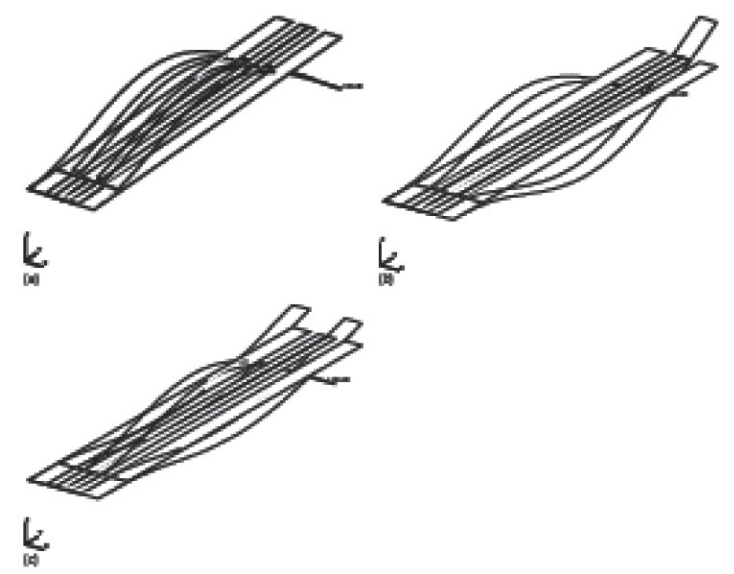

$4^{\text {th }}, 5^{\text {th }}$ and $6^{\text {th }}$ modes
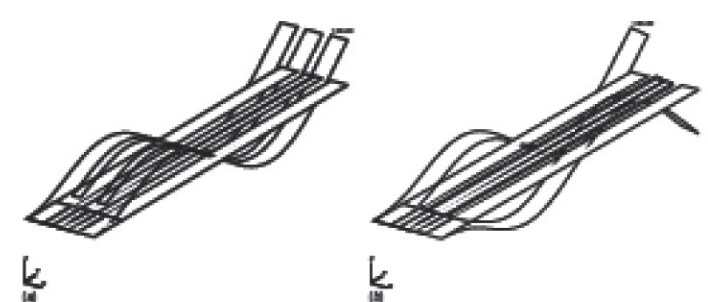

k

k

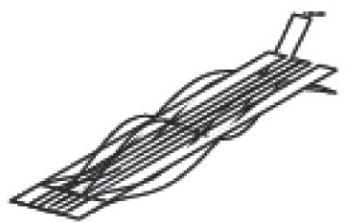

L.

$$
7^{\text {th }}, 8^{\text {th }} \text { and } 9^{\text {th }} \text { modes }
$$

Fig. 10 The mode shapes from the $4^{\text {th }}$ to the $9^{\text {th }}$ [7].

The measured natural frequencies and damping factors for the first nine modes are shown in Table 2. The three mode groups were separated according to the type of mode shape: a bending, a torsion and a mixed one. Each mode group consists of three modes.

Table 2 Measured natural frequencies, damping factors and modal types of the clamped plate with slots [7].

\begin{tabular}{|c|c|c|c|c|c|c|c|c|c|}
\hline Mode: & 1 & 2 & 3 & 4 & 5 & 6 & 7 & 8 & 9 \\
\hline$f[\mathrm{~Hz}]$ & 12.46 & 14.36 & 15.01 & 75.60 & 88.94 & 93.97 & 232.74 & 243.37 & 261.93 \\
\hline$\xi[\%]$ & 0.1032 & 0.0969 & 0.1159 & 0.1404 & 0.1389 & 0.1254 & 0.1494 & 0.0953 & 0.1260 \\
\hline Type & $\mathrm{B}$ & $\mathrm{T}$ & $\mathrm{M}$ & $\mathrm{B}$ & $\mathrm{T}$ & $\mathrm{M}$ & $\mathrm{B}$ & $\mathrm{T}$ & $\mathrm{M}$ \\
\hline
\end{tabular}

Mode types B: Bending, T: Torsion, M: Mixed 


\section{IDYALS JOUSSILL \\ of

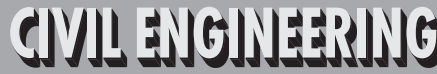

2012/4 PAGES $17-27$

The measured values on Fig. 11a are seen in three different viewing plots. The results of the regression by mode groups on Fig. 11b are shown. The analytical form of the regression function in each group is $f_{1}\left(\omega^{2}\right)=a_{0}+a_{1} \cdot\left(\omega^{2}\right)^{1 / 2}$ with different coefficients of $a_{0}, a_{1}$.

\subsection{Experimental investigation III: Ambient Response Analysis of the Heritage Court Tower Building}

Let us see as an example the regression of the modal damping taken from the literature based on the measurements of Brincker et al. [9].
The modal shapes of the Tower Building were examined. The photo of this building is seen in Fig.12. Its size is $20 \mathrm{~m} \times 25 \mathrm{~m} \times 50 \mathrm{~m}$. The building structure has classical normal modes. The eigenvalue parameters and types of modal shapes are summarized in Table 3. In processing the published data, we got the following results. The damping and eigenfrequency values were plotted on traditional and proposed diagrams as shown in Fig. 13.

For this data series the regression was performed by mode groups as in the previous chapter. One mode group is of the bending modes; the other is of the torsion ones. The results are shown in Fig. 14.
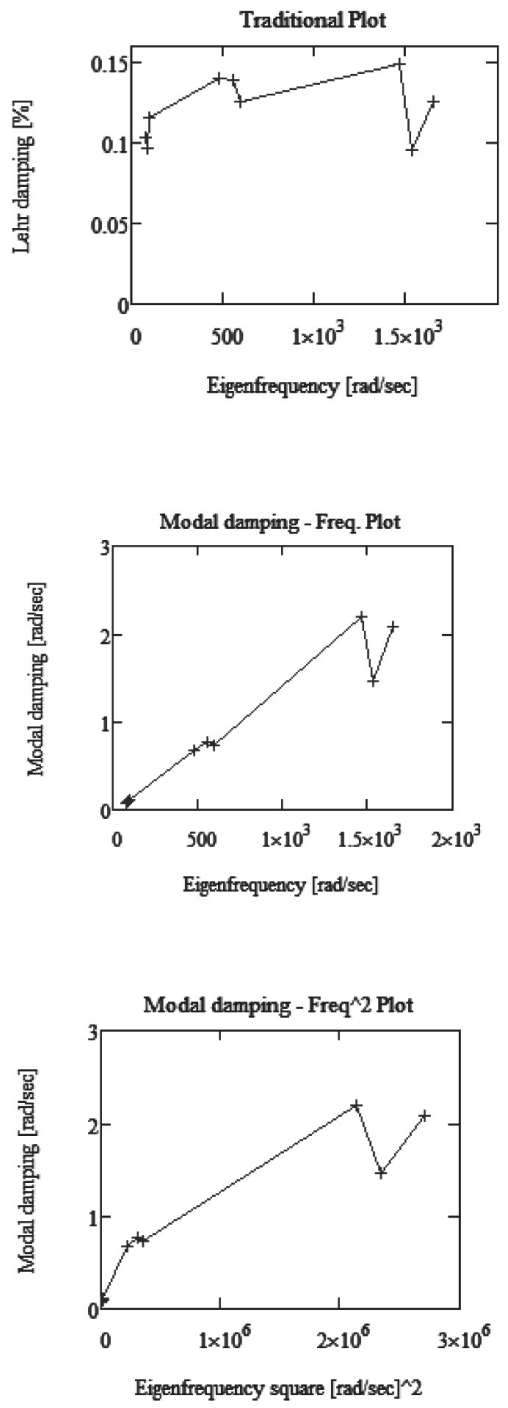

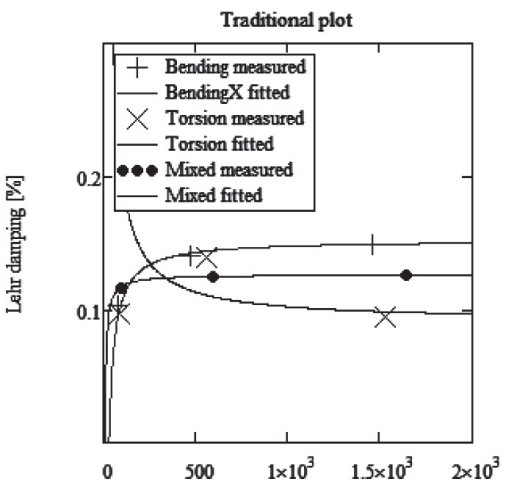

Eigenfrequency [rad/sec] Modal damping - Freq. Plot
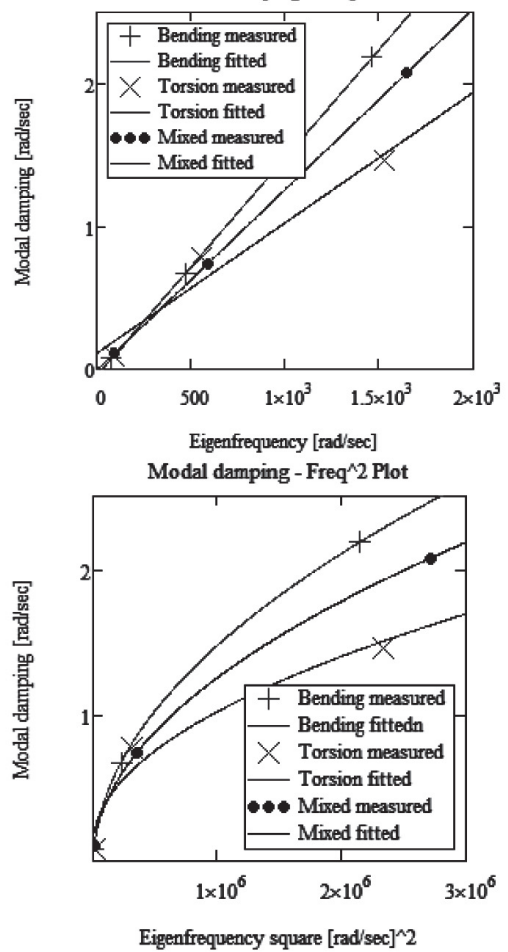

Fig 11. Regression of the eigenvalue parameters of the plate. 


\section{IIOYASS JOUSSIAL \\ O)

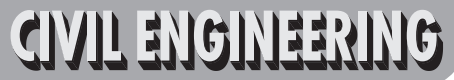

2012/4 PAGES $17-27$

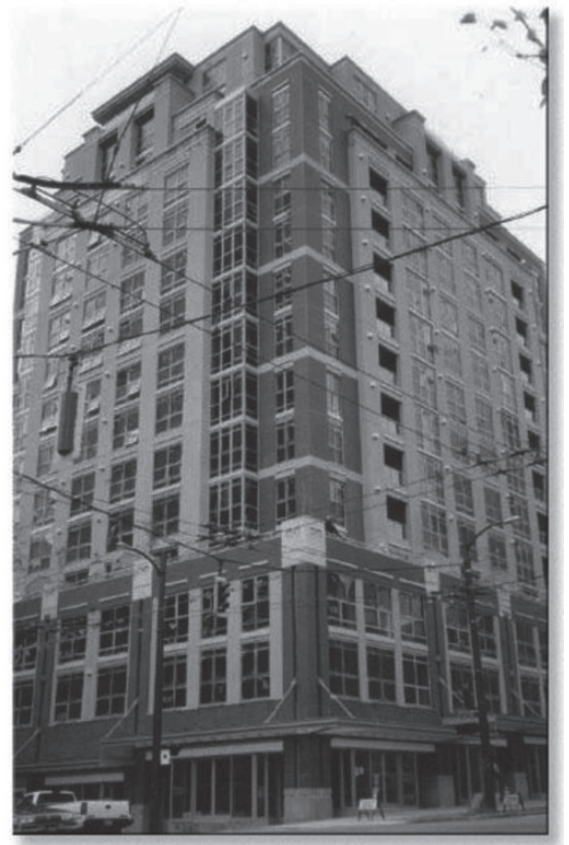

Fig. 12 Picture of the investigated building.
These results raise the following questions:

- Could classical normal modes exist in the case of a not global generator function?

- Can regression be done per-mode-group without the loss of the classical normal modes?

The following section describes the method of grouping the modes.

\section{GROUPING OF THE MODES}

The spectral decomposition of matrix $\mathbf{M}^{-1} \mathbf{K}$, using the expressions in Eqs. (6b) and (6d), is:

$$
\mathbf{M}^{-1} \mathbf{K}=\boldsymbol{\Phi} \boldsymbol{\Omega}^{2} \boldsymbol{\Phi}^{-1}
$$

which is the sum of the n number of dyads (columns of $\boldsymbol{\Phi}$, and rows of $\boldsymbol{\Phi}^{-1}$, and the matrix $\boldsymbol{\Omega}^{2}$ is a diagonal matrix).

Let us make $m$ number of mode groups. According to the mode groups, $m$ performs a partition of the columns of the modalmatrix $\boldsymbol{\Phi}$, the elements of the spectral matrix $\boldsymbol{\Omega}$, and the rows of the inverse of modalmatrix $\boldsymbol{\Phi}^{-1}$,

Table 3 Building's EMA measurement results [9].

\begin{tabular}{|c|c|c|c|c|c|c|c|c|c|c|c|}
\hline Mode i & 1 & 2 & 3 & 4 & 5 & 6 & 7 & 8 & 9 & 10 & 11 \\
\hline$f_{\mathrm{i}}[\mathrm{Hz}]$ & 1.23 & 1.28 & 1.45 & 3.85 & 4.25 & 5.34 & 6.39 & 7.47 & 7.58 & 8.22 & 9.26 \\
\hline$\xi_{\mathrm{i}}[\%]$ & 2.12 & 1.77 & 1.2 & 1.16 & 1.52 & 1.69 & 1.54 & 2.2 & 2.31 & 2.66 & 1.95 \\
\hline Type & BXZ1 & $\mathrm{T} 1$ & BYZ1 & $\mathrm{T} 2$ & $\mathrm{BXZ2}$ & $\mathrm{BYZ2}$ & $\mathrm{T} 3$ & $\mathrm{BXZ}$ & $\mathrm{BXZ}$ & $\mathrm{BXZ}$ & $\mathrm{T} 4$ \\
\hline
\end{tabular}

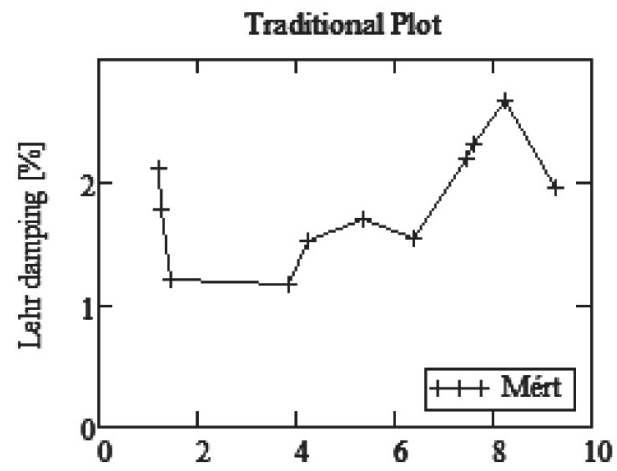

Eigenfrequency [Hz]

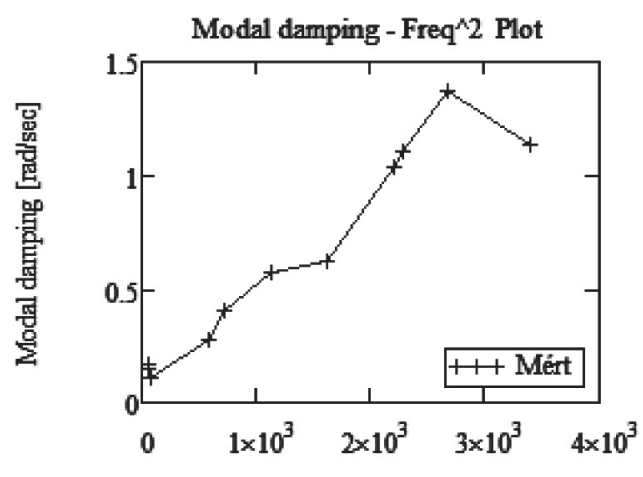

Eigenfrequency square [(rad/sec $\left.)^{\wedge} 2\right]$

Fig. 13 Eigenvalue parameters of the investigated Tower Building [9]. 


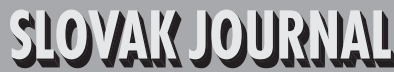 \\ 0,

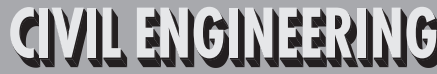

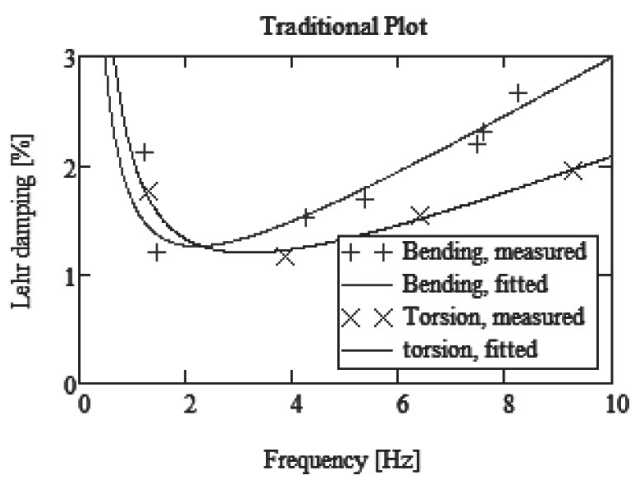

a. Traditional Plot

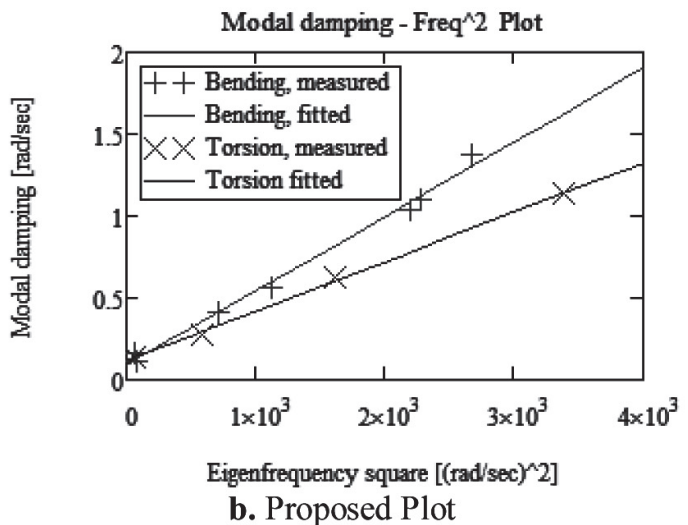

b. Proposed Plot

Fig. 14 Eigenvalue parameters of the Tower Building investigated [9] Regression of damping by mode group.

$$
\begin{aligned}
& \boldsymbol{\Phi}=\left[\begin{array}{llllll}
\boldsymbol{\Phi}_{1} & \boldsymbol{\Phi}_{2} & \ldots & \boldsymbol{\Phi}_{p} & \ldots & \boldsymbol{\Phi}_{m}
\end{array}\right], \boldsymbol{\Phi}^{-1}:=\boldsymbol{\Psi}^{\mathrm{T}}=\left[\begin{array}{c}
\boldsymbol{\Psi}_{1}^{T} \\
\boldsymbol{\Psi}_{2}^{T} \\
\vdots \\
\boldsymbol{\Psi}_{p}^{T} \\
\vdots \\
\boldsymbol{\Psi}_{m}^{T}
\end{array}\right], \\
& \boldsymbol{\Omega}=\left[\begin{array}{llllll}
\boldsymbol{\Omega}_{1} & & & & & \\
& \boldsymbol{\Omega}_{2} & & & & \\
& & \ddots & & & \\
& & & \boldsymbol{\Omega}_{p} & & \\
& & & \ddots & \\
& & & & \boldsymbol{\Omega}_{m}
\end{array}\right]
\end{aligned}
$$

where $\boldsymbol{\Phi}_{p}$ are the eigenvectors of mode group $p ; \Psi_{p}^{T}$ are the row vectors of $\boldsymbol{\Phi}^{-1}$, which belong to mode group $p$, and finally $\boldsymbol{\Omega}_{p}$ are the elements of the spectral matrix belonging to mode group $p$. The spectral decomposition of $\mathbf{M}^{-1} \mathbf{K}$ in terms of mode groups is

$$
\mathbf{M}^{-1} \mathbf{K}=\sum_{p=1}^{m} \boldsymbol{\Phi}_{p} \boldsymbol{\Omega}_{p}^{2} \boldsymbol{\Psi}_{p}^{T} .
$$

Let functions $f_{p}($.$) be the regression functions of the mode groups.$ It is verifiable that the damping matrix

$$
\mathbf{C}=\mathbf{M} \cdot \sum_{p=1}^{m} f_{p}\left(\boldsymbol{\Phi}_{p} \boldsymbol{\Omega}_{p}^{2} \boldsymbol{\Psi}_{p}^{T}\right)
$$

generated by functions $f_{p}($.$) satisfies the commutative relationship$ in Eq. (4), so the damped system has classical normal modes, the proof is seen in the Appendix. Using $\boldsymbol{\Psi}_{p}^{T}=\boldsymbol{\Phi}_{p}^{T} \cdot \mathbf{M}$ based on Eq. (6b) in Eq. (22), we can obtain an explicit form of the classical damping matrix

$$
\mathbf{C}=\mathbf{M} \cdot \sum_{p=1}^{m} \boldsymbol{\Phi}_{p} \cdot f_{p}\left(\boldsymbol{\Omega}_{p}^{2}\right) \cdot \boldsymbol{\Phi}_{p}^{T} \cdot \mathbf{M}
$$

(see Lancaster damping matrix).

If the regression function is not global, but the grouping of the modes is secure, you can use the following procedure and application field:

1. The experimental modal analysis is carried out in the investigated frequency range, and the damping and eigenfrequency values are identified.

2. The eigenfrequency-damping values are plotted. The dampingeigenfrequency points are grouped according to the types of modal shapes.

3. The regression functions are fitted on points by the group of modes.

4. The fitted smooth continuous functions used as extrapolation functions can give the value of the damping for the outband modes, which can be used with FEM programs to input the damping values for the unmeasured modes.

5. The generalized proportional damping matrix can be made in an explicit form by applying Eq. (23).

\section{SUMMARY, ADDITIONAL TASKS}

In this paper the possibilities of identifying damping mechanisms have been reviewed. After our performed measurements and an analysis of the literature, the main conclusions are: 
Construction of a damping matrix by a global generator function is not always possible.

However, it may be constructed by functions defined in mode groups, without the loss of classical normal modes.

\section{Additional tasks}

The grouping of modes is should be solved. Such a method should be developed by which the classification of a modal shape can be performed automatically. The effects that cause variations in the damping values with an EMA method should be analysed.

\section{Acknowledgment}

F.P. acknowledges the support of the New Széchenyi Plan Új

Széchenyi Terv TÁMOP-4.2.1/B-09/1/KMR-2010-0002.

\section{REFERENCES}

[1] Caughey, T. K., O'Kelly, M. E. J.: Classical Normal Modes in Damped Linear Dynamic System. Transaction of the ASME. Journal of Applied Mechanics 32, 1965. pp:583-588.

[2] Caughey, T. K.: Classical Normal Modes in Damped Linear Dynamic System. Transaction of the ASME. Journal of Applied Mechanics June. 1960. pp:269-271.

[3] Fawzy, T. K.: A Theorem on the Free Vibration of Damped System. Transaction of the ASME. Journal of Applied Mechanics Marc. 1977. pp:132-134.

[4] Sas, P., Ward, H., Lammens, S.: Modal Analysis Theory and Testing. Katholike Universiteit Leuven. ISBN 90-73802-61-X. Heverlee (Belgium) 1998. pp: 1-300.

[5] Adhikari, S.: Damping modelling using generalized proportional damping. Journal of Sound and Vibration. 2006, Vol. 293, Nos. 1-2, pp: 156-170.

[6] Rózsa, P.: Linear Algebra and Applications. Műszaki könyvkiadó, Budapest, 1976. p. 685 (in Hungarian)

[7] Adhikari, S., Phani, S.: Experimental Identification of Generalized Proportional Viscous Damping Matrix. Transaction of the ASME Journal of Vibration and Acoustics. February 2009, Vol. 131, pp:1-12.

[8] Pápai, F.: Structural Diagnostic of Building and Materials Handling Machines by Application of Experimental Modal Analysis. Budapest University of Technology and Economics. Faculty of Transportation Engineering. Department of Building
Machines, Materials Handling Machines and Manufacturing Logistic. PhD Thesis, 2007. p. 179, (in Hungarian).

[9] Brincker, R., Andersen, P.: Ambient Response Analysis of the Heritage Court Tower Building Structure, IMAC XVIII: Proceedings of the International Modal Analysis Conference (IMAC), San Antonio, USA, February 7-10, 2000, pp. 1081-1087.

[10] Benedettini, F., Gentile, C.: Operational Modal Testing and FE Model Tuning of Cable-stayed Bridge. Engineeering Structures (2011)

[11] Allen, M. S.: Global and Multi-Input-Multi-Output (MIMO) Extension of the Algorithm of Mode Isolation (AMI). PhD Thesis, 2005, Georgia Institute of Technology.

[12] Siringoringo, D. M., Fujino, Y.: System Identification of Suspension Bridge from Ambient Vibration Response. Engineering Structures 30 (2008) 462-477

[13] Doebling, S. W., Farrar, C. R., Goodman, R. S.: Effects of Measurement Statistics on the Detection of Damage in the Alamosa Canyon Bridge. Proc. of the 15th International Modal Analysis Conference, Orlando, FL, Feb. 3-6, 1997, pp. 919-929.

[14] Cunha, A., Caetano, E., Brincker, R. - Andersen, P.: Identification from the Natural Response of Vasco da Gama Bridge. Proceedings of IMAC-22 : A Conference on Structural Dynamics, January 26 - 29, 2004, Hyatt Regency Dearborn, Dearborn, Michigan, USA. pp. 202-209. 


\section{IDYALS JOUSSILL \\ 0:

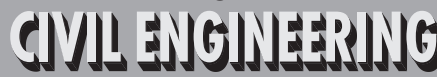

2012/4 PAGES $17-27$

\section{APPENDIX}

\section{Theorem:}

The damping matrix generated by

$$
\mathbf{C}=\mathbf{M} \cdot \sum_{p=1}^{m} f_{p}\left(\boldsymbol{\Phi}_{p} \boldsymbol{\Omega}_{p}^{2} \boldsymbol{\Psi}_{p}^{T}\right)
$$

satisfies the commutative relationship.

\section{Proof:}

Consider the $\left(\mathbf{M}^{-1} \mathbf{C}\right) \cdot\left(\mathbf{M}^{-1} \mathbf{K}\right)=\left(\mathbf{M}^{-1} \mathbf{K}\right) \cdot\left(\mathbf{M}^{-1} \mathbf{C}\right)$ commutative relationship. Substitute the spectral decomposition of all the matrices in it. The left side of this relationship is

$\left.\sum_{p=1}^{m} f_{p}\left(\boldsymbol{\Phi}_{p} \boldsymbol{\Omega}_{p}^{2} \boldsymbol{\Psi}_{p}^{T}\right)\right) \cdot\left(\sum_{q=1}^{m} \boldsymbol{\Phi}_{q, i} \boldsymbol{\Omega}_{q}^{2} \boldsymbol{\Psi}_{q}^{T}\right)=\left(\sum_{p=1}^{m} \boldsymbol{\Phi}_{p} f_{p}\left(\boldsymbol{\Omega}_{p}^{2}\right) \boldsymbol{\Psi}_{p}^{T}\right) \cdot\left(\sum_{q=1}^{m} \boldsymbol{\Phi}_{q} \boldsymbol{\Omega}_{q}^{2} \boldsymbol{\Psi}_{q}^{T}\right)=\sum_{p=1}^{m} \sum_{q=1}^{m} \boldsymbol{\Phi}_{p} f_{p}\left(\boldsymbol{\Omega}_{p}^{2}\right) \boldsymbol{\Psi}_{p}^{T} \boldsymbol{\Phi}_{q} \boldsymbol{\Omega}_{q}^{2} \boldsymbol{\Psi}_{q}^{T}$.

The right side of this relationship is:

$$
\left(\mathbf{M}^{-1} \mathbf{C}\right)=\left(\sum_{q=1}^{m} \boldsymbol{\Phi}_{q, i} \boldsymbol{\Omega}_{q}^{2} \boldsymbol{\Psi}_{q}^{T}\right) \cdot\left(\sum_{p=1}^{m} f_{p}\left(\boldsymbol{\Phi}_{p} \boldsymbol{\Omega}_{p}^{2} \boldsymbol{\Psi}_{p}^{T}\right)\right)=\left(\sum_{q=1}^{m} \boldsymbol{\Phi}_{q} \boldsymbol{\Omega}_{q}^{2} \boldsymbol{\Psi}_{q}^{T}\right) \cdot\left(\sum_{p=1}^{m} \boldsymbol{\Phi}_{p} f_{p}\left(\boldsymbol{\Omega}_{p}^{2}\right) \boldsymbol{\Psi}_{p, j}^{T}\right)=\sum_{q=1}^{m} \sum_{p=1}^{m} \boldsymbol{\Phi}_{q} \boldsymbol{\Omega}_{q}^{2} \boldsymbol{\Psi}_{q}^{T} \boldsymbol{\Phi}_{p} f_{p}\left(\boldsymbol{\Omega}_{p}^{2}\right) \boldsymbol{\Psi}_{p}^{T}
$$

Here

$$
\boldsymbol{\Psi}_{q}^{T} \boldsymbol{\Phi}_{p}= \begin{cases}\mathbf{E}, & \text { if } q=p \\ \mathbf{O}, & \text { if } q \neq p\end{cases}
$$

Consequently,

$$
\begin{aligned}
& \left(\mathbf{M}^{-1} \mathbf{C}\right) \cdot\left(\mathbf{M}^{-1} \mathbf{K}\right)=\sum_{p=1}^{m} \boldsymbol{\Phi}_{p} f_{p}\left(\boldsymbol{\Omega}_{p}^{2}\right) \boldsymbol{\Omega}_{p}^{2} \boldsymbol{\Psi}_{p}^{T}, \\
& \left(\mathbf{M}^{-1} \mathbf{K}\right) \cdot\left(\mathbf{M}^{-1} \mathbf{C}\right)=\sum_{p=1}^{m} \boldsymbol{\Phi}_{p} \boldsymbol{\Omega}_{p}^{2} f_{p}\left(\boldsymbol{\Omega}_{p}^{2}\right) \boldsymbol{\Psi}_{p}^{T} .
\end{aligned}
$$

The right side of Eq. (A-2) equals the right side of Eq. (A-3), because a $f_{p}\left(\mathbf{\Omega}_{p}^{2}\right)$ and $\boldsymbol{\Omega}_{p}^{2}$ are diagonal matrices, so they are commutative. Consequently, the left side of Eq. (A-2) and the left side of Eq. (A-3) are equal, so the commutative relationship is satisfied. 